\title{
Barbed vs Polyglactin 910: A Comparative Study of the Efficacy in Laparoscopic Vaginal Cuff Closure
}

\author{
Prathap Talwar ${ }^{1}$, Lakshmi Velayudam², Hema PL Kukreja ${ }^{3}$, Soumya R Patil ${ }^{4}$
}

\begin{abstract}
Context: Total laparoscopic hysterectomy (TLH) is a popular mode of hysterectomy in the recent times. One of the principal steps is vaginal cuff closure, with many variations in surgical technique and materials. Intracorporeal suturing and knot-tying are crucial steps and are considered to be the most technically difficult skills. To overcome these challenges and learning curve, various measures have been emerging. One among them is the introduction of barbed suture, a new class of suture material.

Aim: To evaluate whether the use of barbed suture for vaginal cuff closure during TLH reduced the surgical difficulty and suturing time when compared to polyglactin 910 suture.

Materials and methods: This randomized comparative study included 100 patients divided into two groups of 50 each, who underwent TLH with vault closure using either barbed sutures or polyglactin 910. Demographic details, indication for surgery, intraoperative complications, mean suturing time, surgeon difficulty, and average hospital stay were compared between the two groups.

Statistical analysis: Student $t$ test for continuous variables and Fischer exact test for categorical variables. $p$ values $\leq 0.05$ were considered significant.

Results: Use of barbed suture has significantly reduced the suturing time for vaginal vault closure ( 5.39 vs 6.9 minutes, $p$ value $<0.0001)$ as well as the technical difficulty in laparoscopic suturing ( $p$ value $<0.0001)$ when compared to that with polyglactin 910.

Conclusion: The introduction of barbed sutures for vault closure during TLH not just reduces the suturing time but is also technically less demanding, making it a potential asset in laparoscopic hysterectomies.

Keywords: Barbed suture, Polyglactin 910, Total laparoscopic hysterectomy, Vault closure.

World Journal of Laparoscopic Surgery (2020): 10.5005/jp-journals-10033-1415
\end{abstract}

\section{INTRODUCTION}

Hysterectomy is the most frequently performed gynecological surgery in the world. ${ }^{1}$ This procedure can be performed through abdominal, vaginal, or laparoscopic access. Besides, robotic-assisted laparoscopy and single-port hysterectomy have been developed in the recent years.

Harry Reich performed the first laparoscopic hysterectomy in 1988 in Pennsylvania. ${ }^{2}$ Since then, laparoscopic hysterectomy has proven to be a safer choice than traditional surgery for benign gynecological conditions. The progress in the field of minimally invasive surgery has transformed laparoscopic hysterectomy into most popular mode of hysterectomy in the recent times due to its cosmetic superiority, shorter hospital stay, quicker resumption of day-to-day activities, and reduced morbidity. ${ }^{3}$

In total laparoscopic hysterectomy, there are significant variations in the vaginal cuff closure with respect to mode of suturing, suturing technique, and suture materials used. Vaginal vault closure is done by intracorporeal sutures or transvaginal sutures, by continuous or interrupted sutures, and in single or double layers using knotted or unknotted stitches. ${ }^{4,5}$ Although widely used, conventional sutures may become loose or entangled, requiring constant traction by an assistant or the operating surgeon, all of which may cause instrument collision and tissue tearing leading to prolongation of suturing. ${ }^{6}$ Laparoscopic intracorporeal closure has several advantages, such as longer postoperative vaginal length, minimizing granulation tissue as the vault margins are not everted into the vagina and provide an excellent vault support by incorporating the pericervical ring. ${ }^{7}$ To prevent vaginal
${ }^{1-4}$ Department of Obstetrics and Gynaecology, JSS Medical College and Research Institute, Mysuru, Karnataka, India

Corresponding Author: Soumya R Patil, Department of Obstetrics and Gynaecology, JSS Medical College and Research Institute, Mysuru, Karnataka, India, Phone: +91 9739667595, e-mail: soumyarpatil999@ gmail.com

How to cite this article: Talwar P, Velayudam L, Kukreja HPL, et al. Barbed vs Polyglactin 910: A Comparative Study of the Efficacy in Laparoscopic Vaginal Cuff Closure. World J Lap Surg 2020;13(3): 113-116.

Source of support: Nil

Conflict of interest: None

vault dehiscence, the knots performed laparoscopically should be as safe as the traditional approach.

However, intracorporeal suturing and knot tying are considered to be the most technically difficult skills. The fundamentals of a perfect knot-tying demands easiness, rapid execution, tight knot, easily reproducible steps, and, also very importantly, the type of suture material used. Performing this laparoscopically is most challenging and necessitates adequate skill and experience.

Therefore, it becomes essential to seek a more convenient technique and safer surgical material to overcome the learning curve required for intracorporeal suturing. One such has been the introduction of barbed sutures, which maintain the tensile strength evenly along the entire length of the wound and reapproximates tissue without the use of surgical knot. The favorable results obtained in several studies suggest that the suture material has the 
potential to become an asset in gynecological surgery. The purpose of this study is to compare the efficacy of barbed suture with that of Polyglactin 910 for vaginal cuff closure in patients undergoing total laparoscopic hysterectomy (TLH) at our hospital.

\section{Materials and Methods}

This comparative study includes 100 patients who underwent total laparoscopic hysterectomy during a period of 12 months. Considering 1-year statistics at our hospital for laparoscopic hysterectomies, i.e., 147 and compensating dropouts, 100 was taken as the sample size, with 50 in each group.

$$
\begin{aligned}
S & =Z^{2} \mathrm{pq} / d^{2} \\
& =1.96 \times 1.96 \times 0.08 \times 0.92 / 0.05 \times 0.05 \\
& =113
\end{aligned}
$$

Following approval by the institutional ethics committee, patients diagnosed with benign gynecological conditions were included and those excluded were malignancies, infected masses, and immunocompromised status. The patients were selected by simple random sampling to avoid bias and were divided into two groups (50 each).

All patients were admitted 4 to 6 hours before surgery after detailed preoperative workup. TLH in both groups were performed by the same surgeon following standard surgical technique. After removal of the uterus, needles were introduced and the vaginal vault closed with continuous intracorporeal sutures using Barbed suture No.1/Polyglactin 910 No.1, where suture was taken starting from the right side through vaginal angle incorporating the right uterosacral, anteriorly through vaginal mucosa followed by posterior vaginal mucosa up till the left uterosacral and left vaginal angle. Then, the needles were removed through the peripheral trocars.

Intraoperatively, mean suturing time, defined as the time taken from beginning of the first stitch and cutting of the last stitch, was noted and compared between the two groups. The amount of blood loss was recorded intraoperatively. The difficulty perceived by the surgeon in operating was graded using a visual analog scale (VAS) ranging from 1 (low difficulty) to 10 (high difficulty). Incidence of intraoperative complications were noted. Postoperatively, the duration of hospital stay was noted. All patients were advised sexual abstinence and avoid heavy lifting of weights for 6 weeks after surgery.

Statistical analysis was carried out using Student $t$ test for continuous variables and Fischer exact test for categorical variables. $p$ values $\leq 0.05$ were considered significant.

\section{Results}

A total of 100 women undergoing total laparoscopic hysterectomy were studied, among which 50 intracorporeal vaginal cuff closures were performed using polyglactin 910 sutures (group I), while another 50 women (group II) were sutured with barbed sutures. Comparison between demographic characteristics is listed in Table 1.

Mean age of women in group I was 45.74 years (SD $=4.96$ years) and that in group II was 44 years (SD $=6.29$ years) without significant differences in age. There was no significant difference in BMI between the two groups, although 6 patients belonged to the obese category with $\mathrm{BMI}$ in the range of 30 to $34.9 \mathrm{~kg} / \mathrm{m}^{2}$.
Most common medical comorbidity noted among the patients was diabetes mellitus $(n=4)$ followed by hypertension $(n=3)$. Among 27 patients with a history of previous abdominal surgery, 19 (70\%) patients had undergone LSCS and 5 patients had a history of appendicectomy. No statistical significance was noted between the two groups in terms of medical comorbidity and previous abdominal surgery.

Table 2 depicts the indication of surgery in both groups. Uterine fibroid was the most common indication for surgery in both the groups followed by endometrial hyperplasia.

The average time taken for suturing vaginal vault was 6.9 minutes ( $\mathrm{SD}=1.27$ minutes) while using polyglactin 910 suture, whereas in group II where barbed suture was used, the suturing time was 5.39 minutes ( $S D=0.76$ minutes) with a significant $p$ value of $<0.0001$. Significant reduction in the difficulty of operation was noted while using barbed sutures for vault closure. The degree of surgical difficulty was lower in the group using barbed sutures (VAS of 3.5 vs $8 ; p$ value $<0.001$ ) (Table 3 ).

There were three intraoperative complications reported of which two cases had bladder injury due to previous LSCS and one case had a rectal serosal injury due to dense endometriotic adhesions. These were not related to the suturing technique but were related to the surgical difficulty due to adhesions. All patients were discharged on the second postoperative day other than the patients with intraoperative complications who stayed longer for further management.

\section{Discussion}

Hysterectomies have been performed vaginally, abdominally, or with laparoscopic or robotic assistance. Laparoscopic hysterectomy has many proven benefits over the other traditional methods, such as shorter hospital stays, faster resumption of routine activities, lower intraoperative blood loss, and fewer wound infections. ${ }^{3}$ However, longer operative duration and higher rates of complications, such as secondary hemorrhage, lower urinary tract injuries, and vaginal cuff dehiscence, have been reported more in laparoscopic than abdominal hysterectomy probably

Table 1: Demographic and clinical details of 100 patients

\begin{tabular}{llll}
\hline & $\begin{array}{l}\text { Polyglactin 910 } \\
\text { group }(n=50)\end{array}$ & $\begin{array}{l}\text { Barbed group } \\
(n=50)\end{array}$ & p value \\
\hline Age (years), mean (SD) & $45.74(4.96)$ & $44(6.29)$ & 0.12 \\
$\mathrm{BMI}\left(\mathrm{kg} / \mathrm{m}^{2}\right)$, mean (SD) & $26.77(2.20)$ & $26.45(2.02)$ & 0.44 \\
Medical comorbidity & $5(10 \%)$ & $7(14 \%)$ & 0.5 \\
$\begin{array}{l}\text { Previous abdominal } \\
\text { surgery } n(\%)\end{array}$ & $17(34 \%)$ & $10(20 \%)$ & 0.1 \\
\hline
\end{tabular}

Table 2: Indications for hysterectomy

\begin{tabular}{lll}
\hline & $\begin{array}{l}\text { Polyglactin 910 } \\
\text { group }(n=50)\end{array}$ & $\begin{array}{l}\text { Barbed group } \\
(n=50)\end{array}$ \\
\hline Fibroid uterus & $26(52 \%)$ & $20(40 \%)$ \\
Endometrial hyperplasia & $10(20 \%)$ & $6(12 \%)$ \\
Adenomyosis & $7(14 \%)$ & $6(12 \%)$ \\
Endometrial polyp & $2(4 \%)$ & $12(24 \%)$ \\
Endometriosis & $3(6 \%)$ & $4(8 \%)$ \\
PID & $0(0 \%)$ & $2(4 \%)$ \\
Fibroid with endometriosis & $1(2 \%)$ & $0(0 \%)$ \\
Chronic cervicitis & $1(2 \%)$ & $0(0 \%)$ \\
\hline
\end{tabular}


Table 3: Mean suturing time

\begin{tabular}{llll}
\hline & $\begin{array}{l}\text { Polyglactin 910 } \\
\text { group }(n=50)\end{array}$ & $\begin{array}{l}\text { Barbed suture } \\
\text { group }(n=50)\end{array}$ & p value \\
\hline $\begin{array}{l}\text { Suturing time (minute), } \\
\text { mean (SD) }\end{array}$ & $6.91(1.27)$ & $5.39(0.76)$ & $<0.0001$ \\
$\begin{array}{l}\text { Degree of surgical } \\
\text { difficulty (VAS) }\end{array}$ & $8.16(0.77)$ & $3.18(0.85)$ & $<0.0001$ \\
$\begin{array}{l}\text { Intraoperative } \\
\text { complications } \\
\begin{array}{l}\text { Duration of hospital } \\
\text { stay (days) }\end{array}\end{array}$ & $1(2 \%)$ & $2(4 \%)$ & 0.6 \\
\hline
\end{tabular}

$\mathrm{SD}=$ Standard deviation

due to increased use of thermal energy by electrocoagulation. ${ }^{8-11}$ These limitations are mostly due to longer learning curve required for laparoscopic procedures as well as for laparoscopic closure of vaginal vault. ${ }^{9}$

In laparoscopic surgeries, the surgeon enters the body cavity through a small incision and operates with a limited range of motion. The endpoints of the instruments move in the opposite direction to the movement of the surgeon's hands making the procedure laborious and difficult to learn. In addition to this, the proximity to vital anatomical structures and the limitation in gaining direct access to it in case of an emergent situation adds on to the complexity of laparoscopic procedures. ${ }^{12}$ A recent survey done by Weizman et al. ${ }^{13}$ suggested that the key factor limiting laparoscopic surgery includes laparoscopic suturing along with other technical and practical limitations. Laparoscopic intracorporeal suturing remains one of the most challenging and time-consuming tasks for surgeons, with the primary reason for this being the need to tie the knots in a confined space with limited visibility.

Suturing during vaginal cuff closure is considered a challenging step in laparoscopic hysterectomy, and the surgical difficulties can result in vault complications such as vaginal cuff dehiscence. Uccella et al. reported a higher incidence of vaginal cuff dehiscence $(0.64 \%)$ for laparoscopic when compared to open transvaginal cuff closure (0.18\%). Probable reason for this is that the magnified view during laparoscopic procedure causes the surgeon to involve less tissue and tension in closure. ${ }^{14}$

Although widely used, conventional sutures carry the drawbacks of requirement for tying knots for anchorage, need to maintain constant tension on the suture which requires traction by the operating surgeon or an assistant, leading to prolongation of suturing. Thus, it becomes essential to simplify intracorporeal laparoscopic suture, knotting skills and reduce the relative technical requirements. Numerous strategies were undertaken, one such has been the introduction of barbed sutures.

Barbed sutures are absorbable sutures with a surgical needle at one end and an annular coil component at the other end. This suture self-anchors at approximately every $1 \mathrm{~mm}$ of tissue, resulting in an evenly distributed tensile strength along the total length of the wound without the need for tying knots. The presence of tiny barbs spaced evenly in a helical array require less technical skill for performing swift suturing and less time than conventional suturing. ${ }^{15}$

The first use of barbed sutures in gynecologic surgery was reported in 2008 by Greenberg and Einarsson. ${ }^{15}$ Since then it has been used in procedures such as laparoscopic myomectomy, hysterectomy as well as re-anastomosis of fallopian tubes and sacro-colpopexies.
In the present study, we observed a significant decrease in time required for vaginal vault closure with the use of barbed suture compared to polyglactin 910 suture. Kim et al. ${ }^{16}$ compared V-Loc $(n=64)$ and Vicryl sutures $(n=106)$ for laparoscopic vaginal cuff closure and they reported a significant reduction in vaginal cuff closure time (7.2 minutes, SD: 1.2 minutes for V-Loc and 12.2 minutes, SD: 3.3 minutes for Vicryl; $p<0.0001$ ) which is consistent with the finding of this study.

Similar results were observed in a single-port total laparoscopic hysterectomy done by Song and Lee, where laparoscopic suturing was adopted for cuff closure in both the groups with experimental group using V-Loc suture (43 cases) and control group applying conventional laparoscopic vaginal cuff suture (59 cases). The V-Loc suture group not only dramatically decreased vaginal stump suturing time (11.4 vs 22.5 minutes; $p$ value $<0.001)$ and total operation time (92 minutes vs 105.2 minutes; $p$ value $=0.002$ ) but also reported reduced difficulty in suture procedure. ${ }^{17}$

Furthermore, a randomized trial by Alessandri et al. comparing unidirectional barbed suture with the traditional continuous suture for laparoscopic myomectomy found that the time required to suture the uterine wall defect and intraoperative blood loss was much less while using barbed sutures. ${ }^{18}$ Barbed sutures have significantly reduced the time required for suturing and the degree of surgical difficulty in a randomized clinical study by Ardovino et al. ${ }^{19}$ comparing the feasibility and safety of barbed bidirectional sutures vs standard sutures for vaginal cuff closure following total laparoscopic hysterectomy and lymph node dissection for early endometrial cancer.

Generally, for gynecologists, transvaginal suturing is widely preferred, as it is technically easier and has shorter learning curve. However, statistical analysis suggests that in TLH procedure, barbed sutures used in vaginal cuff closure reduced the suturing duration as well as technical difficulty experienced by the surgeon, which is in accordance with the above-mentioned literature reports.

With the introduction of a new technology, complications will invariably arise. One of the rare yet potentially serious complication from the use of barbed suture is bowel obstruction. If the cut end of the barbed suture is left long, it may become attached to the overlying bowel or mesentery producing kinking and acting as a transition point of obstruction. Rombaut et al. reported a case of bowel obstruction due to bidirectional suture causing terminal ileal strangulation following laparoscopic myomectomy. ${ }^{20}$ In another case report by Thubert et al., the patient was diagnosed 1 month after undergoing laparoscopic sacrocolpopexy with peritoneal closure using a barbed suture, with small bowel volvulus and mesenteric rupture. ${ }^{21}$ However, there were bowel complications among patients in both groups in the present study.

\section{Conclusion}

In conclusion, this study demonstrated that the use of barbed sutures for laparoscopic vaginal vault closure reduces the suturing time as well the operative difficulty. Based on the results and literature, the use of barbed sutures is an efficient alternative to conventional sutures for laparoscopic vaginal vault closure.

\section{References}

1. Begum M, Zulfiqar N, Yasmin F. Total laparoscopic hysterectomy: a two-year experience in Apollo hospitals Dhaka. Pulse 2016;8(1):21-29. DOI: 10.3329/pulse.v8i1.28097. 
2. Lin C, Li C, Wei B, et al. V-Loc ${ }^{\mathrm{TM}} 180$ suture in total laparoscopic hysterectomy: a retrospective study comparing polysorb to barbed suture used for vaginal cuff closure. Eur J Obstet Gynecol Reprod Biol 2016;207:18-22.

3. Wright JD, Herzog TJ, Tsui J, et al. Nationwide trends in the performance of inpatient hysterectomy in the United States. Obstet Gynecol 2013;122(2, PART 1):233-241. DOI: 10.1097/AOG.0b013e318299a6cf.

4. Moustafa $M$, Elnasharty $M$. Issues around vaginal vault closure. Obstetric Gynaecolog 2019;21(3):203-208. DOI: 10.1111/tog.12573.

5. Blikkendaal MD, Twijnstra ARH, Pacquee $\mathrm{SCL}$, et al. Vaginal cuff dehiscence in laparoscopic hysterectomy: influence of various suturing methods of the vaginal vault. Gynecol Surg 2012;9(4): 393-400. DOI: 10.1007/s10397-012-0745-5.

6. Shah HN, Nayyar R, Rajamahanty S, et al. Prospective evaluation of unidirectional barbed suture for various Indications in surgeoncontrolled robotic reconstructive urologic surgery: wake forest university experience. Int Urol Nephrol 2012;44(3):775-785. DOI: 10.1007/s11255-011-0075-y.

7. David JB, Lindsay M, Janet R, et al. Total laparoscopic hysterectomy: Our 5-year experience (1998-2002). Ochsner J 2010;10(1):8-12.

8. Paul PG, Prathap T, Kaur H, et al. Secondary hemorrhage after total laparoscopic hysterectomy. JSLS 2014;18(3):e2014.00139. DOI: 10.4293/JSLS.2014.00139.

9. Uccella S, Ceccaroni M, Cromi A, et al. Vaginal cuff dehiscence in a series of 12,398 hysterectomies: effect of different types of colpotomy and vaginal closure. Obstet Gynecol 2012;120(3):516-523. DOI: 10.1097/AOG.0b013e318264f848.

10. Paul PG, Panditrao AS, Khan S, et al. Secondary haemorrhage after different modes of hysterectomy. Gynecol Surg 2013;10(4):267-272. DOI: 10.1007/s10397-013-0811-7.

11. Clarke-Pearson DL, Geller EJ. Complications of hysterectomy. Obstet Gynecol 2013;121(3):654-673. DOI: 10.1097/AOG.0b013e3182841594.

12. Puri V, Patel A, Majumder $\mathrm{K}$, et al. Intraoperative conversion from video-assisted thoracoscopic surgery lobectomy to open thoracotomy: a study of causes and implications. J Thorac Cardiovasc Surg 2015;149(1):55-61. DOI: 10.1016/j.jtcvs.2014.08.07462.e1.

13. Weizman NF, Maurer R, Einarsson Jl, et al. Survey on barriers to adoption of laparoscopic surgery. J Surg Educ 2015;72(5):985-994. DOI: 10.1016/j.jsurg.2015.04.001.

14. Uccella S, Ghezzi F, Mariani A, et al. Vaginal cuff closure after minimally invasive hysterectomy: our experience and systematic review of the literature. Am J Obstet Gynecol 2011;205(2):119.e1-12. DOI: 10.1016/j. ajog.2011.03.024.

15. Greenberg. JA. The use of barbed sutures in obstetrics and gynaecology. Rev Obstet Gynecol 2010;3:82-91.

16. Kim JH, Byun SW, Song JY, et al. Barbed versus conventional 2-layer continuous running sutures for laparoscopic vaginal cuff closure. Medcine 2016;95(39):39. DOI: 10.1097/MD.0000000000004981.

17. Song T, Lee SH. Barbed suture vs traditional suture in single port total laparoscopic hysterectomy. J Minim Invasive Gynecol 2014;21(5): 825-829. DOI: 10.1016/j.jmig.2014.03.012.

18. Alessandri F, Remorgida V, Venturini PL, et al. Unidirectional barbed suture versus continuous suture with intracorporeal knots in laparoscopic myomectomy: a randomized study. J Minim Invasive Gynecol 2010;17(6):725-729. DOI: 10.1016/j.jmig.2010. 06.007.

19. Mario A, Maria AC, Fernando F, et al. Bidirectional barbed suture in total laparoscopic hysterectomy and lymph node dissection for endometrial cancer: Technical evaluation and 1-year follow-up of 61 patients. J Laparoendosco Adv Surg Techniq 2013;23(4):347-350. DOI: 10.1089/lap.2012.0079.

20. Rombaut S, Baulies S, Cusido M, et al. Quill barbed suture-related complication. Gynecol Surg 2012;9(3):359-361. DOI: 10.1007/s10397012-0749-1.

21. Thubert T, Pourcher G, Deffieux X. Small bowel volvulus following peritoneal closure using absorbable knotless device during laparoscopic sacral colpopexy. Int Urogynecol J 2011;22(6):761-763. DOI: 10.1007/s00192-010-1348-1. 